\title{
BMJ Open Feasibility of Improving Cerebral Autoregulation in Acute Intracerebral Haemorrhage (BREATHE-ICH) study: a protocol for an experimental interventional study
}

Jatinder S Minhas, ${ }^{1}$ Ronney B Panerai, ${ }^{1,2}$ Thompson G Robinson ${ }^{1,2}$

To cite: Minhas JS, Panerai RB, Robinson TG. Feasibility of Improving Cerebral Autoregulation in Acute Intracerebral Haemorrhage (BREATHE-ICH) study: a protocol for an experimental interventional study. BMJ Open 2018;8:e020758. doi:10.1136/ bmjopen-2017-020758

- Prepublication history for this paper is available online. To view these files, please visit the journal online (http://dx.doi. org/10.1136/bmjopen-2017020758).

Received 21 November 2017 Revised 29 January 2018 Accepted 6 March 2018
Check for updates

${ }^{1}$ Cerebral Haemodynamics in Ageing and Stroke Medicine (CHiASM) Research Group, Department of Cardiovascular Sciences, University of Leicester, Leicester, UK

${ }^{2}$ National Institute for Health Research Leicester Biomedical Research Centre, University of Leicester, Leicester, UK

Correspondence to Dr Jatinder S Minhas; jm591@le.ac.uk

\section{ABSTRACT}

Introduction Cerebral autoregulation (CA) is impaired in a multitude of neurological conditions. Increasingly, clinical studies are correlating the nature of this impairment with prognostic markers. In acute intracerebral haemorrhage $(\mathrm{ICH})$, impairment of $\mathrm{CA}$ has been associated with worsening clinical outcomes including poorer Glasgow Coma Score and larger haematoma volume. Hypocapnia has been shown to improve CA despite concerns over hypoperfusion and consequent ischaemic risks, and it is therefore hypothesised that hypocapnia (via hyperventilation) in acute ICH may improve CA and consequently clinical outcome. BREATHE-ICH is a CAtargeted interventional study in acute $\mathrm{ICH}$ utilising a simple bedside hyperventilatory manoeuvre.

Methods and analysis Patients with acute ICH within 48 hours of onset will be included. The experimental setup measures cerebral blood flow (cerebral blood velocity, transcranial Doppler), blood pressure (Finometer) and end tidal carbon dioxide (capnography) at baseline, and in response to hypocapnia $(-5 \mathrm{~mm}$ and $-10 \mathrm{~mm} \mathrm{Hg}$ below baseline) achieved via a 90 s hyperventilatory manoeuvre. Autoregulation is evaluated with transfer function analysis and autoregulatory index calculations. Important classical endpoints associated with this before and after interventional study include death and disability at 14 days and the proportion of recruited individuals able to comply with the full measurement protocol.

Ethics and dissemination A favourable opinion was granted by the East Midlands-Nottingham 1 Research Ethics Committee (17/EM/0283). It is anticipated that the results of this study will be presented at national and international meetings, with reports being published in journals during late 2018.

Trial registration number NCT03324321.

\section{INTRODUCTION}

The ability to maintain an adequate brainblood flow despite sudden changes in blood pressure (BP) can be measured in an individual and is referred to as the process of 'autoregulation'. 1 This control process has been shown to be impaired in acute
Strengths and limitations of this study

- The study set-up carefully considers risks of hypoperfusion during the manoeuvre in a vulnerable clinical neurological circumstance.

- Although participants recruited to the study are likely to be mild to moderate stroke severity, as evidenced by neurological impairment (National Institutes of Health Stroke Scale 5-12) and Glasgow Coma Score $>8$ in similar experimental studies, this study is designed to assess feasibility and provide important mechanistic insights into the impact of a novel cerebral autoregulation (CA)-targeted intervention.

- The proposed study investigates for the first time whether a CA-targeted intervention involving a simple breathing exercise in survivors of acute intracerebral haemorrhage is safe, feasible and effective in improving $\mathrm{CA}$ and subsequently clinical outcomes.

ischaemic stroke (AIS), head injury, in dementia or following premature birth, but importantly is associated with worse patient outcomes. $^{2}$ An Autoregulation Index (ARI) can be assigned between 0 and 9 ( 0 being poor and 9 being the most efficient cerebral autoregulation (CA) observed) to gauge how good the control over cerebral blood flow $(\mathrm{CBF})$ is at a given time. ${ }^{3}$ Dynamic CA (dCA) is a measure of the response of $\mathrm{CBF}$ to rapid changes in BP, and several key studies have shown impaired dCA postacute intracerebral haemorrhage (ICH) ${ }^{4-8}$ Recent studies have demonstrated that dCA impairment lasts up to 12 days, ${ }^{78}$ and that CA is bilaterally disturbed after supratentorial $\mathrm{ICH}^{8}$ Importantly, the most recent study showed larger haematoma volume is likely to independently predict poorer CA status ipsilateral to haematoma. ${ }^{8}$

Spontaneous acute ICH is associated with both high mortality and morbidity. ${ }^{9}$ There 
is a relative paucity of management options for acute ICH compared with AIS with BP control the foremost approach. However, controversy exists as to whether intensive BP lowering in acute ICH risks cerebral ischaemia. This is particularly the case if CBF control mechanisms are altered by chronic hypertension or indeed ICH itself. CA provides a protective mechanism for the brain parenchyma from extremes of CBF change, with the risk of hyperperfusion or hypoperfusion in response to systemic BP changes. A key limitation of large-scale randomised controlled trials has been the ability to provide mechanistic insight into CBF during the acute phase of haemorrhagic stroke. ${ }^{10}$ Lower mean CBF, in combination with impaired CA, may have implications for more intensive $\mathrm{BP}$ lowering and warrants further studies examining such strategies on CBF regulatory mechanisms.

Hypocapnia has been historically used in the management of acute brain injury to reduce intracranial pressure (ICP) and to improve outcome in specific circumstances. ${ }^{11} 12$ This is usually weighed against the risk of hypoperfusion and subsequent neuronal ischaemia, which could worsen outcome. Arterial carbon dioxide tension $\left(\mathrm{PaCO}_{2}\right)$ is a balance between production and removal. ${ }^{12}$ In order to generate hypocapnia, this usually involves a hyperventilatory manoeuvre often in the intubated patient. The use of hypocapnia in acute brain injury has been primarily focused around the scientific principles of the Monro-Kellie doctrine describing the brain as a 'closed box' with a fixed volume. ${ }^{11}$ Raised ICP (sustained $>20 \mathrm{~mm} \mathrm{Hg}$ ) can cause secondary brain injury by impairing cerebral perfusion, direct pressure or by brainstem herniation. ${ }^{12}$

Hypocapnia can be induced to lower ICP by decreasing the $\mathrm{CBF}$ via cerebral arterial vasoconstriction. The effects can be potent: CBF decreases by approximately $3 \%$ per $\mathrm{mm} \mathrm{Hg}$ change in $\mathrm{PaCO}_{2}$ (range, 60-20 mm Hg $\mathrm{PCO}_{2}$ ) in patients with traumatic brain injury (TBI) ${ }^{11}{ }^{13}$ Despite advances in our understanding of $\mathrm{PaCO}_{2}$ changes in TBI and other circumstances associated with raised ICP, little is understood about the use of hyperventilation in acute stroke. Hyperventilation has classically been advocated in acute stroke for two reasons: first to reduce ICP, and second to restore homeostasis to penumbral zones around ischaemic tissue by inducing inverse steal and correcting acidosis. ${ }^{12}$ These physiological changes are weighed against both potential vasoconstriction leading to hypocapnia-induced brain ischaemia as well as cerebral hyperaemia due to subsequent normalisation of $\mathrm{PaCO}_{2}{ }^{12}$ Lastly, cerebral ischaemic lesions noted on MRI in patients with acute ICH also raise the possibility of harms associated with BP lowering which may be more relevant than issues of perihaematomal ischaemia.

Hitherto, randomised studies have only been conducted in patients with TBI, with benefits noted in sustained ICP below $25 \mathrm{~mm} \mathrm{Hg}$, but with a less favourable outcome in those with moderate motor impairment on their Glasgow Coma Score (GCS) ${ }^{1415}$ However, this population is very different from acute stroke patient populations, which form the focus of this study.

This method of manipulating dCA may improve CA in acute ICH; offering a novel non-pharmacological intervention, particularly as hypocapnia (induced by hyperventilation), has been used as a neuroprotective mechanism improving impaired dCA in rats with subarachnoid haemorrhage ${ }^{16}$ in patients with liver failure, ${ }^{17}$ and patients undergoing isoflurane anaesthesia ${ }^{18}$ and acute bacterial meningitis. ${ }^{19}$

Importantly, hypocapnia decreases CBF but expands the plateau region of the cerebral autoregulatory curve, hence improving CA and subsequently the ability to keep $\mathrm{CBF}$ constant for a wide range of perfusion pressures. ${ }^{2021}$ Manning et al demonstrated systolic BP variability confers a poor outcome in acute ICH and concluded that peaks in systolic BP are likely to confer harm, as opposed to smooth and sustained early intensive lowering (to $140 \mathrm{~mm} \mathrm{Hg}$ ) which is likely to be beneficial. ${ }^{22}$ However, with a growing body of evidence highlighting impaired CA as a marker of poor prognostic outcomes in acute ICH, this relationship is likely to hold significant importance in possible alternative interventions for this devastating neurological state. The argument therefore for assessing the feasibility of hypocapnia for improving CA in acute ICH rests on mild to moderate hypocapnia possibly being of greater clinical benefit than methods that are designed to alter the magnitude of CBF. Therefore, the study will assess if: (1) ICH sufferers will tolerate the hyperventilation manoeuvre; (2) hyperventilation manoeuvre leads to hypocapnia in the ICH population; (3) dCA will be improved, while maintaining safe levels of CBF. The proposed study investigates for the first time whether a CA-targeted intervention involving a simple breathing exercise in survivors of acute ICH is safe, feasible and effective in improving CA and subsequently clinical outcomes. If successful we hope to take this simple bedside intervention forward to design a larger randomised controlled trial.

\section{METHODS AND ANALYSIS \\ Sample selection}

Patients with acute ICH, recruited within 48 hours of onset, able to comply with a respiratory manoeuvre (hyperventilation).

Each patient with stroke will be required to participate for up to 14 days poststroke symptom onset, during which up to a total of two assessments will be made. The first assessment will be recorded while the participant is an inpatient under the care of the University Hospitals of Leicester NHS Trust Stroke Service (acute: within 48 hours of symptom onset); the next assessment will be within 10-14 days poststroke onset (subacute).

The sample size included is based on the investigators' pilot study, which included 45 healthy volunteers who underwent the hyperventilation manoeuvre outlined in the 'Intervention' section (Minhas et al, unpublished data). 
Formal sample size calculations have been previously conducted. In order to detect a change of 1 unit in ARI, and to calculate the required sample size we followed the technique as previously described by others. ${ }^{23}$ For this study, a minimum sample of 40 patients provides $80 \%$ power at the $5 \%$ significance level to detect a difference in ARI of 1 unit.

The inclusion and exclusion criteria were determined based on the clear need for the recruited individual to be able to engage with the instructions associated with the manoeuvre to ensure as reliable a physiological measurement as possible.

\section{Inclusion criteria}

- clinical diagnosis of haemorrhagic stroke within 48 hours of onset (for patients waking with a stroke, time of onset will be taken to be the time when the patient was last asymptomatic);

- able and willing to give informed consent;

- male or female, aged 18 years or above;

- able (in the investigator's opinion) and willing to comply with all study requirements;

- willing to allow his or her general practitioner to be notified of participation in the study.

\section{Exclusion criteria}

- male or female, aged below 18 years;

- significant previous airway disease (formal diagnosis of moderate or severe airway disease and having treatment for this respiratory condition-via inhalers or specialist input);

- unable (in the investigator's opinion) or unwilling to comply with any study requirements;

- female participants who are pregnant, lactating or planning pregnancy during the course of the study;

- clinical diagnosis of stroke greater than 48 hours from onset;

- having had a resolved transient ischaemic attack (ie, neurological symptoms completely resolved upon hospital presentation);

- comorbidity with anticipated life expectancy less than 3 months.

\section{Intervention}

The level of impairment of dCA will be compared with extent of CT acute ICH findings. dCA measurements will be measured as below with a hyperventilation protocol. This will involve sustained periods of $90 \mathrm{~s}$ of hyperventilation at two levels $(-5 \mathrm{~mm} \mathrm{Hg}$ and $-10 \mathrm{~mm} \mathrm{Hg}$ below baseline end tidal carbon dioxide $\left(\mathrm{EtCO}_{2}\right)$ ) regulated using a metronome. The patients will receive a demonstration from the conducting physician clarifying the expected relationship of their breathing with the metronome beat. Two-minute washout periods of normal respiration will be allowed between successive measurements. Each incremental reduction in $\mathrm{PCO}_{2}$ will be repeated on two occasions during the same session.
Participants will be monitored throughout the process and any unexpected physiological changes will be recorded on an adverse event form. These will be gathered and discussed on a monthly basis with a clinician independent from the study team within our institution (to replicate a data safety monitoring process). Furthermore, individuals will be compared with age-matched controls from the Leicester Cerebral Haemodynamics Database $^{2}$ and the pilot study to ensure physiological parameters are within acceptable limits (Minhas et al, unpublished data). Furthermore, in preparation for the study, lower limits of acceptable $\mathrm{EtCO}_{2}(29 \mathrm{~mm} \mathrm{Hg})$ and cerebral blood velocity (CBV; $33 \mathrm{~cm} / \mathrm{s})$ will be calculated as within $1 \mathrm{SD}$ from the mean hypocapnia level for cohort of healthy volunteer values and based on previous acceptable limits in TBI studies. ${ }^{24}$ This will help protect individuals from risk of ischaemia associated with hypocapnia-induced vasoconstriction.

\section{Interpretation}

Important classical endpoints associated with this before and after interventional study include death and disability at 14 days (modified Rankin Scale) and the proportion of recruited individuals able to comply with the full measurement protocol. Furthermore, the data will be assessed for quality during the analysis protocol and rejected data files recorded with associated reasoning. Lastly, the percentage change in $\mathrm{CBV}$ at baseline and in response to a hypocapnic manoeuvre in the acute phase $(<48$ hours) and subacute phase (10-14 days) will be recorded alongside ARI. Importantly, this study is designed to test the feasibility of a CA-targeted intervention using hyperventilation. This inclusion of clinical outcomes is not necessarily the main objective of the study but highly relevant as an early indicator of feasibility to support design of robust larger trials. In conclusion, understanding the percentage of patients able to comply with the manoeuvre and whether preservation of autoregulation in this population is possible will provide a strong basis for future work.

\section{Data collection and integration}

For all subjects, all assessments will be undertaken in a dedicated cardiovascular research laboratory at Leicester Royal Infirmary, which is at a controlled temperature $\left(20-24^{\circ} \mathrm{C}\right)$ and is free from distraction or on the hyperacute stroke ward using the mobile physiological measurement equipment (figure 1) if unable to transfer to the laboratory. The subject will lay supine on an examination couch. Baseline casual BP will be calculated as a mean of three supine brachial BP readings using a validated UA767 $\mathrm{BP}$ monitor. Beat-to-beat non-invasive BP will be recorded continuously using the Finometer cuff device attached to the middle finger of the non-dominant hand (non-hemiparetic hand in patients with stroke). R-R interval will be recorded using a 3-lead ECG. Respiratory rate will be recorded, and end tidal $\mathrm{PCO}_{2}\left(\mathrm{EtCO}_{2}\right)$ monitored using small nasal cannulae placed at the base of the nose (Salter Labs, ref 4000) attached to a capnograph (Capnocheck 


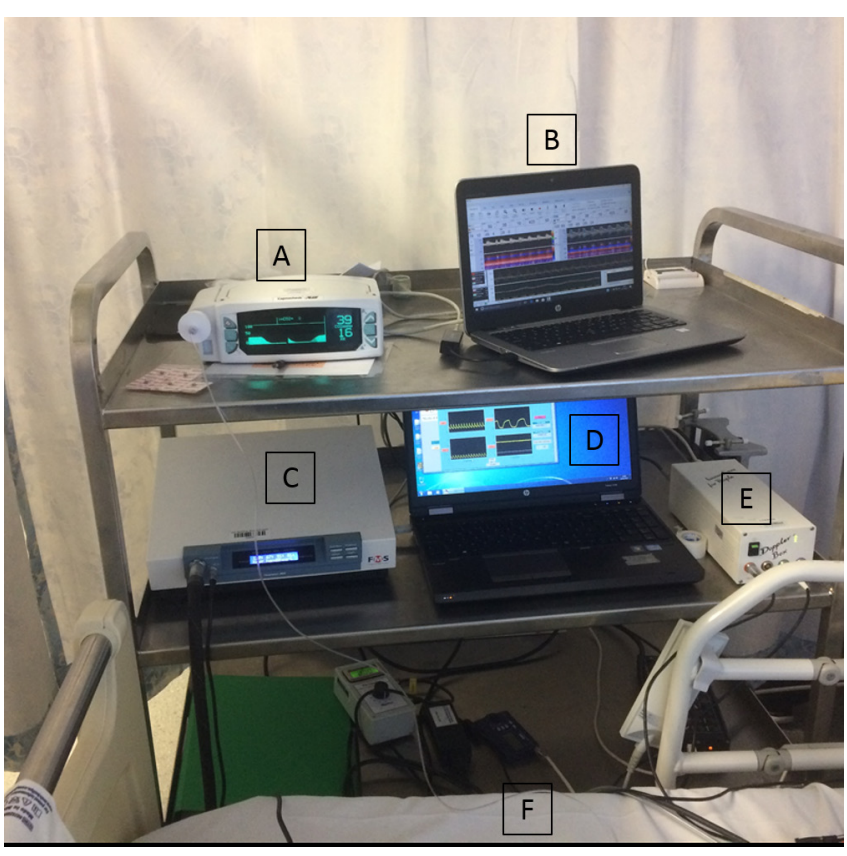

Figure 1 Bedside physiological measurement set-up for BREATHE-ICH study: (A) capnograph; (B) cerebral blood velocity waveforms from Dopplerbox (V.10.5.1 software) dedicated laptop; (C) Finometer; (D) PHYSIDAS data acquisition system; (E) DWL Dopplerbox; (F) Korg Metronome.

Plus) to monitor respiration. Simultaneous bilateral insonation of the middle cerebral arteries (MCA) will be performed with the subject lying supine on a couch using transcranial Doppler ultrasound (TCD, DWL Dopplerbox V.10.5.1 software) to measure $\mathrm{CBV}$ as the most widely accepted surrogate of CBF in dCA studies. ${ }^{21} \mathrm{Using} 2 \mathrm{MHz}$ probes, supported by a custom-made frame, the vessel will be located via the temporal bone window, and identified as the MCA by the waveform, its depth, velocity and the direction of flow. All parameters will be simultaneously recorded onto a computer data acquisition system (PHYSIDAS) for subsequent off-line analysis. The physiological measurement set-up is demonstrated in figure 1. A CT scan within 48 hours will be analysed for haematoma size, perihaematomal oedema and intraventricular extension. The assessment of these imaging parameters will be done blinded to any autoregulation data from initial CT or MRI according to the ABC criteria (assess the length, width and depth of haemorrhage in centimetres) associated with the shape of the haemorrhagic lesion (ABC/2 or $\mathrm{ABC} / 3$ for regular or irregularly shaped haemorrhage, respectively). ${ }^{25}$

\section{Data analysis}

Following transfer function analysis (TFA), a measure of dCA, ARI, will be calculated. TFA is the most widely used and validated technique of examining the relationship between $\mathrm{CBF}$ and BP in the frequency domain. TFA allows quantification of the close transfer of BP fluctuations to $\mathrm{CBF}$, providing an objective measure of dCA. Furthermore, TFA and coherence (linearity between input and output signals) is calculated using Welch's method. Coherence will be used to determine acceptability of good quality data taking into consideration appropriate number of df. ${ }^{26}$ Beyond these parameters, phase and gain are calculated giving an explanation as to the timing of input (BP) and output (CBV) signals. Lastly, to derive ARI, a time domain approach is used as described by Tiecks et al from a standardised set of curves based on $\mathrm{CBV}$ response to sudden step changes in BP following thigh cuff deflation. ${ }^{3}$ A linear mixed model will be fitted to explore the relationship between $\mathrm{EtCO}_{2}$ and the dCA parameter ARI in acute ICH; the variability between subjects will be accounted for by incorporating the patient as a random effect.

\section{Statistical analysis}

All normally distributed continuous variables will be described as mean (SD) and non-Gaussian with skewness as median (IQR). Comparison of baseline data of patients with acute ICH will be made by the Student's t-test for normally distributed data, or by appropriate non-parametric test. Repeated measures analysis of variance (twoway) will be adopted to test for the effect of hypocapnia on CBV and ARI in patients with acute haemorrhagic stroke.

\section{Study limitations}

The study limitations have been carefully considered in the context of current work. First, patients are likely to have mild to moderate stroke severity, as evidenced by neurological impairment (National Institutes of Health Stroke Scale (NIHSS) 5-12) and GCS >8 in similar experimental studies. Furthermore, there is the possibility that in order to comply with the respiratory manoeuvre, patients may have milder stroke severity (NIHSS 1-4). Second, this study will use BP instead of cerebral perfusion pressure to calculate dCA parameters, rather than the more invasive ICP measurement. This is an acceptable approach as we are unlikely to find significantly elevated ICP in this patient population as their neurological status would not permit involvement in the study. Third, we will not be directly assessing the effects of BP-lowering therapy on dCA, so there will remain limited data on the impact of intensive BP lowering on cerebral haemodynamics. Therefore, a mechanistic understanding from a cerebral haemodynamic perspective of different targets for intensive BP lowering is lacking. Fourth, use of nasal cannulae for $\mathrm{PaCO}_{2}$ measurement has benefits associated with rapid response time to changes, though concerns exist over accuracy compared with face mask measurements. ${ }^{27}$ Lastly, with reference to TCD studies, changes in CBF can be accurately expressed by $\mathrm{CBV}$, as long as the diameter of the MCA remains constant. This assumption is usually acceptable at normocapnia or mild hypercapnia, but at moderate levels of hypercapnia, as we achieved in our subjects, it is likely that CBV underestimated CBF. There are no studies to date showing MCA diameter change with hypocapnia despite evidence being shown in hypercapnia. ${ }^{28}$ 


\section{ETHICS AND DISSEMINATION}

\section{Ethics and safety considerations}

The East Midlands-Nottingham 1 Research Ethics Committee (REC) reviewed the BREATHE-ICH study protocol. The committee appraised the ethical implications of the intervention that would be carried out during the study and potential impact on patient's rights and well-being. Favourable opinion from the REC means that due care and consideration has been made to preserve patient's rights and the methodology is ethically justified; favourable opinion was provided on 31 August 2017 (17/EM/0283). Further to this, University Hospitals of Leicester NHS Trust provided local research and innovation approval on 25 September 2017.

Informed consent will be obtained from participants in line with recommendations set out in the E6(R1) of the International Council for Harmonisation of Technical Requirements or Pharmaceuticals for Human Use (ICH) Good Clinical Practice Guidelines.

If the study demonstrates feasibility of improving $\mathrm{CA}$ in acute ICH, the experience and knowledge acquired will support future prospective (randomised) controlled trials.

\section{Dissemination}

We plan to present all data at national and international conferences. Furthermore, we will also publish the findings in international journals. We expect to publish our initial findings during late 2018. Following the publication of our results participant-level data will be available for collaborations, on request.

Contributors JSM, RBP and TGR participated in the developing and testing of the study set-up, conception of the study, designing the statistical and mathematical analysis, and drafted the manuscript.

Funding The BREATHE-ICH study is funded by a Dunhill Medical Trust Research Training Fellowship (RTF97/0117).

Competing interests None declared.

Patient consent Not required.

Ethics approval The East Midlands-Nottingham 1 Research Ethics Committee (17/ $\mathrm{EM} / 0283)$.

Provenance and peer review Not commissioned; externally peer reviewed.

Open Access This is an Open Access article distributed in accordance with the Creative Commons Attribution Non Commercial (CC BY-NC 4.0) license, which permits others to distribute, remix, adapt, build upon this work non-commercially, and license their derivative works on different terms, provided the original work is properly cited and the use is non-commercial. See: http://creativecommons.org/ licenses/by-nc/4.0/

(c) Article author(s) (or their employer(s) unless otherwise stated in the text of the article) 2018. All rights reserved. No commercial use is permitted unless otherwise expressly granted.

\section{REFERENCES}

1. Aaslid R, Lindegaard KF, Sorteberg W, et al. Cerebral autoregulation dynamics in humans. Stroke 1989;20:45-52.

2. Patel N, Panerai RB, Haunton V, et al. The Leicester cerebral haemodynamics database: normative values and the influence of age and sex. Physiol Meas 2016;37:1485-98.
3. Tiecks FP, Lam AM, Aaslid R, et al. Comparison of static and dynamic cerebral autoregulation measurements. Stroke 1995;26:1014-9.

4. Reinhard M, Neunhoeffer F, Gerds TA, et al. Secondary decline of cerebral autoregulation is associated with worse outcome after intracerebral hemorrhage. Intensive Care Med 2010;36:264-71.

5. Nakagawa K, Serrador JM, LaRose SL, et al. Dynamic cerebral autoregulation after intracerebral hemorrhage: A case-control study. BMC Neurol 2011;11:108,2377-11.

6. Oeinck M, Neunhoeffer F, Buttler KJ, et al. Dynamic cerebral autoregulation in acute intracerebral hemorrhage. Stroke 2013;44:2722-8.

7. Ma H, Guo ZN, Liu J, et al. Temporal Course of Dynamic Cerebral Autoregulation in Patients With Intracerebral Hemorrhage. Stroke 2016;47:674-81.

8. Ma H, Guo ZN, Sun X, et al. Hematoma volume is a predictive factor of disturbed autoregulation after spontaneous intracerebral hemorrhage. J Neurol Sci 2017;382:96-100.

9. van Asch CJ, Luitse MJ, Rinkel GJ, et al. Incidence, case fatality, and functional outcome of intracerebral haemorrhage over time, according to age, sex, and ethnic origin: a systematic review and meta-analysis. Lancet Neurol 2010;9:167-76.

10. Anderson CS, Heeley E, Huang Y, et al. Rapid blood-pressure lowering in patients with acute intracerebral hemorrhage. N Engl $J$ Med 2013;368:2355-65.

11. Neumann JO, Chambers IR, Citerio G, et al. The use of hyperventilation therapy after traumatic brain injury in Europe: an analysis of the BrainIT database. Intensive Care Med 2008;34:1676-82.

12. Laffey JG, Hypocapnia KBPN Engl J Med 2002;347:43-53.

13. Brian JE. Carbon dioxide and the cerebral circulation. Anesthesiology 1998;88:1365-86.

14. Muizelaar JP, Marmarou A, Ward JD, et al. Adverse effects of prolonged hyperventilation in patients with severe head injury: a randomized clinical trial. J Neurosurg 1991;75:731-9.

15. Meng L, Gelb AW. Regulation of cerebral autoregulation by carbon dioxide. Anesthesiology 2015;122:196-205.

16. MaX, Willumsen L, Hauerberg J, et al. Effects of graded hyperventilation on cerebral blood flow autoregulation in experimental subarachnoid hemorrhage. J Cereb Blood Flow Metab 2000;20:718-25.

17. Strauss Gl. The effect of hyperventilation upon cerebral blood flow and metabolism in patients with fulminant hepatic failure. Dan Med Bull 2007;54:99-111.

18. McCulloch TJ, Boesel TW, Lam AM. The effect of hypocapnia on the autoregulation of cerebral blood flow during administration of isoflurane. Anesth Analg 2005;100:1463-7.

19. Møller K, Høgh P, Larsen FS, et al. Regional cerebral blood flow during hyperventilation in patients with acute bacterial meningitis. Clin Physiol 2000;20:399-410.

20. Paulson OB, Strandgaard S, Edvinsson L. Cerebral autoregulation. Cerebrovasc Brain Metab Rev 1990;2:161-92.

21. Panerai RB. Assessment of cerebral pressure autoregulation in humans--a review of measurement methods. Physiol Meas 1998;19:305-38.

22. Manning L, Hirakawa $\mathrm{Y}$, Arima $\mathrm{H}$, et al. Blood pressure variability and outcome after acute intracerebral haemorrhage: a post-hoc analysis of INTERACT2, a randomised controlled trial. Lancet Neurol 2014;13:364-73.

23. Brodie FG, Atkins ER, Robinson TG, et al. Reliability of dynamic cerebral autoregulation measurement using spontaneous fluctuations in blood pressure. Clin Sci 2009;116:513-20.

24. Menon DK, Coles JP, Gupta AK, et al. Diffusion limited oxygen delivery following head injury. Crit Care Med 2004;32:1384-90.

25. Huttner HB, Steiner T, Hartmann M, et al. Comparison of ABC/2 estimation technique to computer-assisted planimetric analysis in warfarin-related intracerebral parenchymal hemorrhage. Stroke 2006;37:404-8

26. Claassen JA, Meel-van den Abeelen AS, Simpson DM, et al. Transfer function analysis of dynamic cerebral autoregulation: A white paper from the International Cerebral Autoregulation Research Network. $J$ Cereb Blood Flow Metab 2016;36:665-80.

27. Minhas JS, Robinson T, Panerai R. $\mathrm{PaCO}_{2}$ measurement in cerebral haemodynamics: face mask or nasal cannulae? Physiol Meas 2017;38:N101-N106.

28. Coverdale NS, Gati JS, Opalevych O, et al. Cerebral blood flow velocity underestimates cerebral blood flow during modest hypercapnia and hypocapnia. J Appl Physiol 2014;117:1090-6. 